\title{
Nebulised lipid-polymer hybrid nanoparticles for the delivery of a therapeutic anti-inflammatory microRNA to bronchial epithelial cells
}

\author{
Sebastian Vencken ${ }^{1}$, Camilla Foged ${ }^{2}$, Joanne M. Ramsey ${ }^{3}$, Louise Sweeney ${ }^{4}$, \\ Sally-Ann Cryan ${ }^{3,5,6}$, Ronan J. MacLoughlin ${ }^{4}$ and Catherine M. Greene ${ }^{1}$
}

Affiliations: ${ }^{1}$ Lung Biology Group, Dept of Clinical Microbiology, Royal College of Surgeons in Ireland, Education and Research Centre, Beaumont Hospital, Dublin, Ireland. ${ }^{2}$ Dept of Pharmacy, Faculty of Health and Medical Sciences, University of Copenhagen, Copenhagen, Denmark. ${ }^{3}$ Drug Delivery and Advanced Materials Team and Tissue Engineering Research Group, School of Pharmacy and Dept of Anatomy, Royal College of Surgeons in Ireland, Dublin, Ireland. ${ }^{4}$ Aerogen Ltd, Galway, Ireland. ${ }^{5}$ Trinity Centre for Bioengineering, Trinity College Dublin, Dublin, Ireland. 'SFI Centre for Research in Medical Devices (CURAM), Royal College of Surgeons in Ireland, Dublin and NUI Galway, Galway, Ireland.

Correspondence: Catherine M. Greene, Lung Biology Group, Dept of Clinical Microbiology, Royal College of Surgeons in Ireland, Education and Research Centre, Beaumont Hospital, Dublin 9, Ireland.

E-mail: cmgreenedarcsi.ie

ABSTRACT Modulation of microRNAs (miRNAs), endogenous regulators of gene expression, is a promising strategy for tackling inflammatory lung diseases. In this proof-of-concept study, we tested delivery of miR-17 to bronchial epithelial cells (BECs) using nebulised lipid-polymer hybrid nanoparticles (LPNs). The primary aim was to reduce the induced secretion of miR-17's target, i.e. the pro-inflammatory chemokine interleukin (IL)-8.

Synthetic miR-17 mimics were loaded into LPNs composed of poly(DL-lactic-co-glycolic acid) (PLGA) and the cationic lipid 1,2-dioleoyloxy-3-(trimethylammonium)propane (DOTAP) using a double emulsion solvent evaporation method and nebulised using the Aerogen Solo nebuliser. The physicochemical, aerosol, inflammatory and cytotoxic properties of LPNs were characterised. The effect of LPNs on lipopolysaccharide (LPS)-induced IL-8 production from human NuLi-1 BECs was tested by ELISA.

The $z$-average, polydispersity index and $\zeta$-potential of the LPNs and the aerodynamic properties of nebulised suspensions were in a range optimal for deposition in the bronchi and bronchioles postinhalation. Cytotoxic and pro-inflammatory effects were minimal for LPNs loaded with a model cargo. Nebulisation did not affect the physicochemical or functional properties of the LPNs. Nebulised miR-17loaded LPNs downregulated LPS-induced IL- 8 secretion by $>40 \%$ in BECs.

This study suggests that DOTAP-modified PLGA LPNs are efficient and well-tolerated carriers for delivery of miRNA mimics to BECs.

@ERSpublications

Nebulised miR-17-loaded DOTAP-PLGA nanoparticles are suitable, nontoxic carriers for knockdown of IL-8 in bronchial epithelial cells and form a platform upon which future miRNAbased therapeutics could be developed for inflammatory lung disease http://ow.ly/5iT330nSNZI

Cite this article as: Vencken S, Foged C, Ramsey JM, et al. Nebulised lipid-polymer hybrid nanoparticles for the delivery of a therapeutic anti-inflammatory microRNA to bronchial epithelial cells. ERJ Open Res 2019; 5: 00161-2018 [https://doi.org/10.1183/23120541.00161-2018].

This article has supplementary material available from openres.ersjournals.com

Received: Sept 142018 | Accepted after revision: Feb 192019

Copyright $\odot$ ERS 2019. This article is open access and distributed under the terms of the Creative Commons Attribution Non-Commercial Licence 4.0. 


\section{Introduction}

Localised inflammation of lung tissue is a normal physiological response to respiratory insult by pathogens and airborne particles. However, if the inflammatory response becomes chronic, extensive invasion by immune cells, remodelling of lung tissue over time and narrowing of the airways will eventually result in obstructive lung disease and loss of lung function [1]. Such obstructive inflammatory lung diseases include, for example, chronic obstructive pulmonary disease (COPD), cystic fibrosis (CF), asthma and chronic infectious lung diseases. All can display high baseline inflammation interjected by exacerbations [1].

The airway epithelium is the first line of defence against airborne challenges to the lungs. This epithelium, which includes the bronchial epithelial lining, plays a critical role in the orchestration of the lung inflammatory cascade through its ability to regulate innate and adaptive immune responses [2]. One of the first of these responses is often Toll-like receptor 4-triggered secretion of interleukin (IL)-8 (also known as CXC motif chemokine ligand-8), an important neutrophil chemokine in the lung [3]. Upregulation of IL-8 secretion causes neutrophil infiltration to the site of production $[1,4]$. Chronic secretion of neutrophil-derived proteases and oxidants can damage the lung epithelium and basal tissues, resulting in significant tissue remodelling and a decline of lung function [1,4]. This is an important feature of inflammatory lung diseases [5].

Due to its critical role in lung inflammation, IL- 8 has been investigated as a target for therapy in previous studies that aimed to control chronic inflammatory lung disease [6]. Anti-IL-8 antibodies have been investigated as a potentially effective treatment in induced lung injury models, but subsequent clinical trials with these agents have not yet resulted in approved therapeutics (e.g. ABX-IL8: ClinicalTrials.gov identifier NCT00035828).

Inhalable micro- and nanoparticles have emerged as promising carriers for the delivery of active pharmaceutical ingredients (APIs) directly to the lung epithelium and its underlying basal layers [7]. These APIs include, among others, antisense oligonucleotides that exert their therapeutic effect via the RNA interference (RNAi) pathway by inhibiting the expression of specific proteins involved in disease pathways [7]. Although most efforts to date have been focused on particle-mediated delivery of small interfering RNAs (siRNAs) to the airway epithelium [7-11], the use of microRNA (miRNA) mimics as cargoes may also have significant therapeutic potential. miRNAs are small, endogenous, noncoding RNAs that pleiotropically hybridise to target mRNAs for downregulation through the cell's RNAi machinery, either by target mRNA degradation or by translational repression [12]. Synthetic miRNA mimics have frequently been employed for research purposes, while their possible use in medical treatment is a more recent exploration [13].

We previously showed that miR-17 is a potent inhibitor of IL- 8 and is downregulated in bronchial epithelial cells (BECs) during long-term exposure to inflammatory mediators [14]. In this successive study, we test the ability of nebulised lipid-polymer hybrid nanoparticles (LPNs) to deliver miR-17 to BECs and to knockdown IL-8 expression. For this, we prepared miR-17-loaded LPNs composed of the biodegradable polymer poly(DL-lactic-co-glycolic acid) (PLGA) and the cationic lipid 1,2-dioleoyloxy-3-(trimethylammonium)propane (DOTAP). Nanoparticles based on PLGA have previously been tested for the delivery and sustained release of APIs to the lungs, including pharmaceuticals currently used for the treatment of inflammatory lung diseases such as CF and COPD $[15,16]$.

In this proof-of-concept study, we show that nebulisation facilitates the aerosolisation of DOTAP-modified PLGA LPN suspensions, producing aerosol droplets that are optimal for the delivery of their cargo to the bronchial epithelium. Furthermore, we demonstrate that the LPNs cause minimal cytotoxicity and pro-inflammatory effects. Finally, our data indicate that nebulised miR-17-loaded DOTAP-modified PLGA LPNs can downregulate lipopolysaccharide (LPS)-induced IL-8 secretion in BECs.

\section{Materials and methods}

Preparation of miRNA mimic-loaded DOTAP-modified PLGA LPNs

Double emulsion solvent evaporation was used to encapsulate miR-17 mimics, NC\#1 and double-stranded DNA (dsDNA) oligonucleotides within DOTAP-modified PLGA LPNs as described previously [17, 18]. Additional details are given in the supplementary material.

\section{Physicochemical characterisation}

The $z$-average and polydispersity index (PDI) were measured by dynamic light scattering using photon correlation spectroscopy. The $\zeta$-potential was determined using laser Doppler micro-electrophoresis (Zetasizer Nano ZS; Malvern Instruments, Malvern, UK). For additional details and the measurement of yield, encapsulation efficiency and loading capacity, see the supplementary material. 
Nanoparticle nebulisation

Lyophilised LPN/trehalose cakes were reconstituted in RNase-free water in the absence or presence $0.9 \%$ saline for $10 \mathrm{~min}$ at room temperature followed by gentle vortexing for $3 \mathrm{~min}$. Nebulisation was conducted with the Aerogen Solo vibrating mesh nebuliser (Aerogen, Galway, Ireland). The LPN dispersions were nebulised into a $15 \mathrm{~mL}$ centrifuge tube fixed to the Aerogen Solo and collected after nebulisation by a brief centrifugation for $10 \mathrm{~s}$ at $<500 \times g$. The $z$-average, PDI and $\zeta$-potential before and after nebulisation were measured.

\section{Aerosol characterisation}

The mass median aerodynamic diameter (MMAD) and the geometric standard deviation (GSD) of the nebulised LPN dispersions were measured using the Next-Generation Impactor (NGI; Copley Scientific, Nottingham, UK) at a flow rate of $15 \pm 0.5 \mathrm{~L} \cdot \mathrm{min}^{-1}$. Counting of the nanoparticle fractions was performed with the Nanosight NS300 (Malvern). The volume median diameter (VMD) of the nebulised LPN dispersions and $0.9 \%$ saline was measured using the Spraytec laser diffraction system (Malvern). Additional details are provided in the supplementary material.

\section{Cell culture and treatments}

The human NuLi-1 BEC line (kind donation from Joseph Zabner, University of Iowa, Iowa City, IA, USA) was cultured in collagen-coated plasticware in Bronchial Epithelial Growth Medium (Lonza, Basel, Switzerland) as described previously [19]. In some experiments cells were stimulated for $24 \mathrm{~h}$ with $10 \mu \mathrm{g} \cdot \mathrm{mL}^{-1}$ Pseudomonas aeruginosa LPS. Details of LPN transfections are provided in the supplementary material.

\section{IL-6 and IL-8 ELISA}

IL-8 and IL- 6 concentrations were measured by sandwich ELISA in media removed from cell cultures and centrifuged for $5 \mathrm{~min}$ at $2000 \times g$ at $4^{\circ} \mathrm{C}$ as previously described [3].

Cell viability assay

Cell viability was determined using the CellTiter 96 Aqueous One Solution Cell Proliferation Assay (Promega, Madison, WI, USA). Additional details are provided in the supplementary material.

Caspase-3 activity assay

Apoptotic activity was measured using a caspase-3 activity assay with $50 \mu \mathrm{M}$ DEVD-AMC caspase-3 substrate in $1 \times$ Reaction Buffer (2 mM EDTA, 0.1\% CHAPS (3-((3-cholamidopropyl) dimethylammonio)-1-propanesulfonate), $5 \mathrm{mM}$ dithiothreitol in $20 \mathrm{mM}$ HEPES, $\mathrm{pH} 7.4$ ) as previously described [20].

\section{RNA isolation and miRNA quantitative reverse transcription-PCR}

Total RNA was isolated using the miRNeasy Mini kit (Qiagen, Hilden, Germany). Prior to RNA isolation, special care was taken to exclude uninternalised miRNAs [21]. miR-17 and U6 were quantified with pre-designed reverse transcription and stem-loop PCR primers for miR-17 (assay 002308; Thermo Fisher Scientific) and U6 small nuclear RNA (assay 001973; Thermo Fisher Scientific); reverse transcription (TaqMan MicroRNA Reverse Transcription kit; Thermo Fisher Scientific) and PCR (Universal Master Mix II; Thermo Fisher Scientific) were carried out using the LightCycler 480 System (Roche, Basel, Switzerland).

\section{Statistical analysis}

Continuous variables are presented as mean with standard deviation. Quantitative results were compared using an unpaired or paired t-test, where appropriate. Where multiple hypotheses were tested, Bonferroni correction was applied for multiple comparisons. For regression analysis, both linear and nonlinear goodness-of-fit models were tested, yielding the $\mathrm{R}^{2}$ measure of correlation with testing for a significant deviation from a nonzero slope.

\section{Results}

Physicochemical properties of miRNA mimic- and dsDNA oligonucleotide-loaded LPNs

DOTAP-modified PLGA LPNs loaded with miRNA mimics or dsDNA oligonucleotides were prepared $[17,18]$. Optimal particle size and colloidal stability in suspension is important for efficient delivery of nucleic acid cargoes to the airway epithelium [22-24]. The $z$-average, PDI and $\zeta$-potential were measured immediately after preparation of independent LPN batches by photon correlation spectroscopy and laser Doppler micro-electrophoresis, respectively (table 1 ). The $z$-average of LPNs loaded with miRNA mimics (miR-17 and NC\#1) was $208.0 \pm 16.7 \mathrm{~nm}$ and the PDI was $0.148 \pm 0.030$, which are within target ranges for 


\begin{tabular}{|c|c|c|c|}
\hline Formulation & $\begin{array}{c}z \text {-average } \\
\mathrm{nm}\end{array}$ & PDI & $\begin{array}{l}\text { 丂-potential } \\
\mathrm{mV}\end{array}$ \\
\hline miRNA mimic LPNs (not freeze-dried) & $208.0 \pm 16.7$ & $0.148 \pm 0.030$ & $42.4 \pm 8.4$ \\
\hline dsDNA oligonucleotide LPNs (not freeze-dried) & $200.1 \pm 32.6$ & $0.164 \pm 0.063$ & $35.3 \pm 11$ \\
\hline Lyophilised miRNA mimic LPNs reconstituted ${ }^{\#}$ & $218.5 \pm 5.8$ & $0.195 \pm 0.005$ & $32.6 \pm 6.8$ \\
\hline $\begin{array}{l}\text { Lyophilised miRNA mimic LPNs reconstituted }{ }^{\#} \text { and } \\
\text { nebulised }\end{array}$ & $232.1 \pm 12.9$ & $0.173 \pm 0.007$ & $28.3 \pm 4.4$ \\
\hline \multicolumn{4}{|c|}{$\begin{array}{l}\text { Data are presented as mean } \pm \text { SD. miRNA: microRNA; dsDNA: double-stranded DNA. }{ }^{\#}: 0.9 \% \text { saline was } \\
\text { used for LPN reconstitution. }\end{array}$} \\
\hline
\end{tabular}

efficient mucus penetration and uptake by BECs while avoiding phagocytosis $[22,24]$. The $\zeta$-potential of miRNA-loaded LPNs was $42.4 \pm 8.4 \mathrm{mV}$, which is within a desirable target range to ensure colloidal stability in suspension, minimal toxicity and minimal pro-inflammatory effects [25]. No significant differences between the $z$-average, PDI and $\zeta$-potential were measured for LPNs loaded with miRNA mimics or dsDNA oligonucleotides (table 1; $\mathrm{n}=8$, paired t-test). Reconstitution of freeze-dried LPNs did not affect these properties (table $1 ; n=3$, paired t-test). The average loading capacity of the miRNA mimic-loaded LPNs was $6.96 \pm 2.09 \mu \mathrm{g}$ nucleic acid. $\mathrm{mg}^{-1}$ LPNs. The encapsulation efficiency and yield were $15.5 \pm 4.6 \%$ and $69 \pm 9 \%$, respectively.

\section{Nebulisation of LPNs does not influence their physicochemical properties}

The aerodynamic properties of particles, once nebulised, determine their deposition pattern in the airways. Nanometre-sized particles generally have low deposition efficiency as they fall below the $1-5 \mu \mathrm{m}$ size range that allows sufficient inertia for deposition in the bronchioles [26-28]. To endow LPNs with optimal aerodynamic properties, the LPNs were nebulised in a liquid medium ( $0.9 \%$ saline), resulting in the generation of nanoparticle-loaded liquid droplets of micrometre diameter that can be inhaled.

Independent batches of LPNs loaded with miRNA mimics or dsDNA oligonucleotides were aerosolised with the Aerogen Solo nebuliser. This vibrating mesh nebuliser allows for the gentle dispersion of therapeutics in a liquid medium without imposing significant stress on the formulation that might otherwise compromise chemical and physical stability [29]. To test the quality of aerosolised LPNs their physicochemical properties were compared prior to and after nebulisation. No significant changes in $z$-average, PDI and $\zeta$-potential were evident (table $1 ; \mathrm{n}=3$, paired t-test).

\section{Aerodynamic properties of nebulised LPNs}

To determine the aerosol properties of the nebulised LPNs, the MMAD, which describes the aerodynamic particle size distribution of an aerosol by mass, and the VMD, which reflects the particle size distribution of particles by volume, were measured. The GSD describes the spread of the aerodynamic particle distribution. We first determined that counting LPNs with the Nanosight NS300 was an accurate method for quantifying LPN suspensions collected from the NGI, as it allows for direct counting of the particles in a solution without the need for reference standards (figure 1). The MMAD of LPNs nebulised in $0.9 \%$ saline was $4.20 \pm 0.05 \mu \mathrm{m}$, while the GSD was $1.61 \pm 0.09$. The fine particle fraction (FPF), also called the respirable fraction, was $89.8 \pm 1.7 \%$ at FPF5.4 $\mu \mathrm{m}$. The VMD of the LPNs in $0.9 \%$ saline was $3.71 \pm 0.07 \mu \mathrm{m}$, which showed no significant difference with the VMD of nebulised $0.9 \%$ saline $(3.84 \pm 0.19 \mu \mathrm{m} ; \mathrm{n}=3$, paired t-test).

\section{The PLGA:DOTAP component of LPNs is minimally cytotoxic and pro-inflammatory}

The physicochemical properties (shape, size and charge) of LPNs influence cell viability, and DOTAP-modified PLGA LPNs loaded with chemically modified siRNAs can exhibit cytotoxic and pro-inflammatory effects at high concentrations $[17,30]$. We investigated the impact of the PLGA:DOTAP component of the LPNs on viability and inflammation in NuLi-1 BECs. For this part of the study dsDNA oligonucleotides, which are nontoxic and noninflammatory to NuLi-1 BECs, were encapsulated in LPNs as an alternative to expensive miRNA mimics. The physicochemical properties of dsDNA-loaded LPNs did not differ from those of miRNA-loaded LPNs (table 1). In time-course and dose-response experiments no significant effects on cell viability were observed (figure 2a-c). Caspase-3 activity, a marker for apoptosis, was not increased in $1 \mathrm{mg} \cdot \mathrm{mL}^{-1} \mathrm{LPN}$-treated BECs compared with unexposed control cultures (figure $2 \mathrm{~d}$ ). 
FIGURE 1 Lipid-polymer hybrid nanoparticles (LPNs) counted in suspensions taken from the Next-Generation Impactor (NGI) using the Nanosight NS300. LPNs nebulised through the $\mathrm{NGI}$ were washed from the stages and counted. This was repeated with independent LPN samples. Data are presented as mean \pm SD. $n=3$.
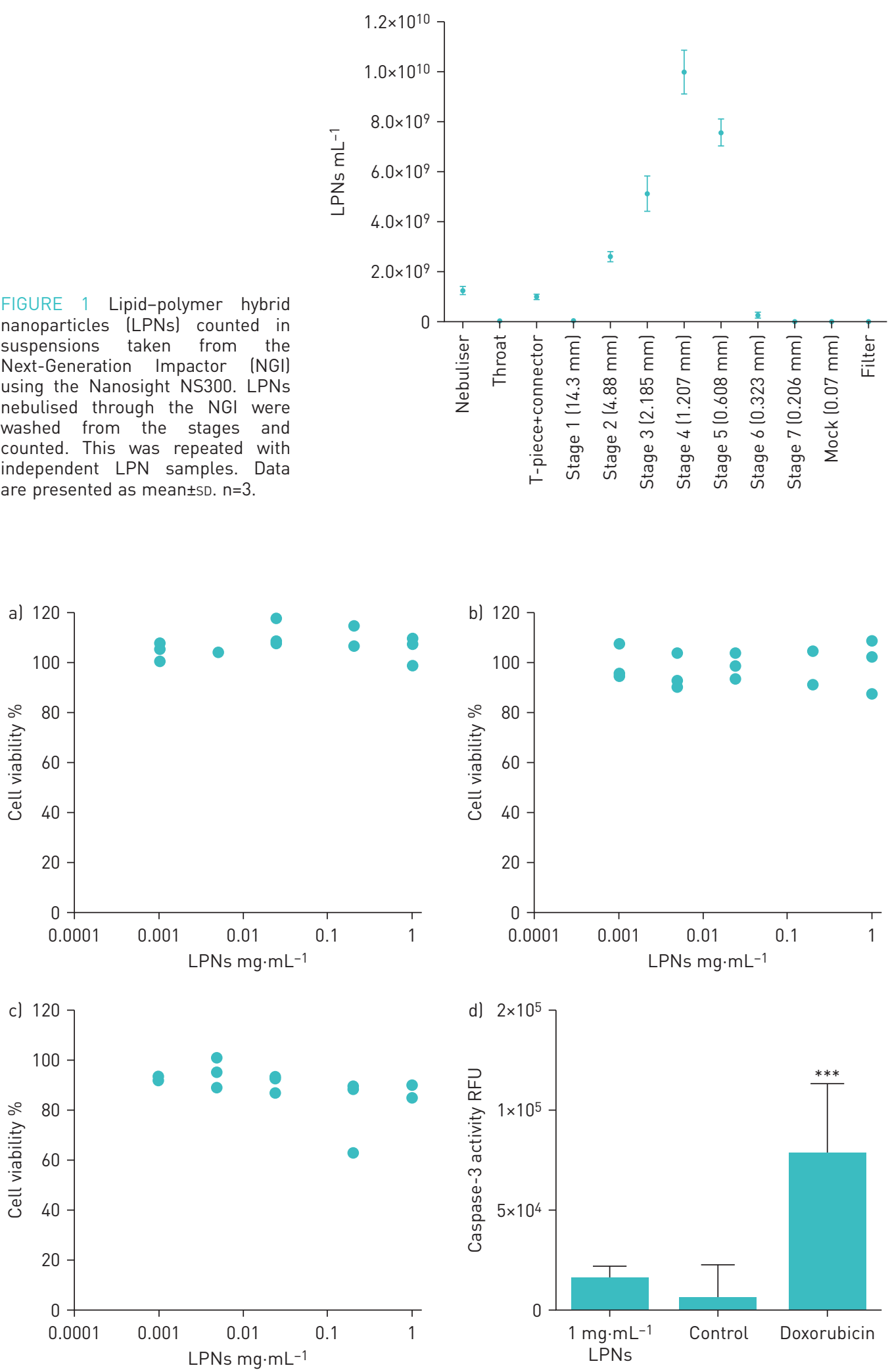

FIGURE 2 Effect of double-stranded DNA (dsDNA) oligonucleotide-loaded lipid-polymer hybrid nanoparticles (LPNs) on NuLi-1 bronchial epithelial cell (BEC) viability. RFU: relative fluorescence units; DOTAP. 1,2-dioleoyloxy-3-(trimethylammonium)propane; PLGA: poly(DL-lactic-co-glycolic acid). a-c) NuLi-1 BECs were exposed to DOTAP-modified PLGA LPNs loaded with dsDNA oligonucleotides as indicated for a) 24, b) 48 and c) $72 \mathrm{~h}$. $\mathrm{n}=3$. d) Caspase- 3 activity in NuLi-1 BECs after $24 \mathrm{~h}$ of exposure to $1 \mathrm{mg} \cdot \mathrm{mL}^{-1}$ DOTAP-modified PLGA LPNs containing dsDNA oligonucleotides, Bronchial Epithelial Growth Medium alone (negative control) or $2 \mu \mathrm{M}$ doxorubicin (positive control). ${ }^{* * *}$ : $\mathrm{p} \leqslant 0.001$, two-tailed t-test. $\mathrm{n}=3$. 

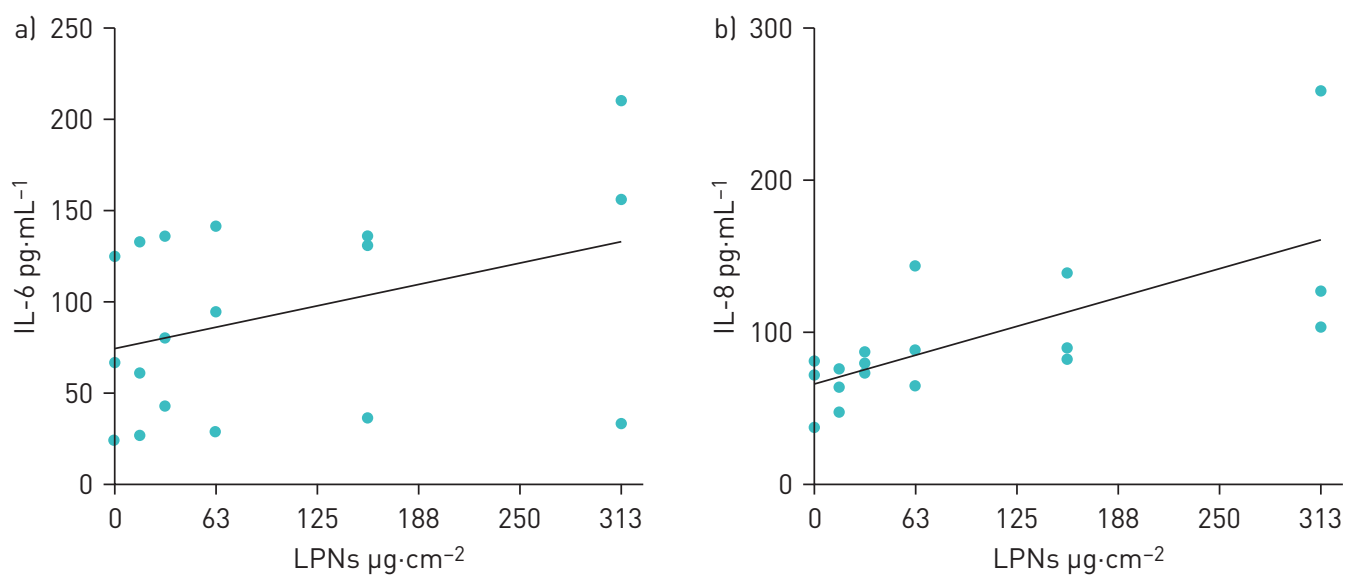

FIGURE 3 Effect of double-stranded DNA (dsDNA) oligonucleotide-loaded 1,2-dioleoyloxy-3-(trimethylammonium) propane (DOTAP)-modified poly(DL-lactic-co-glycolic acid) (PLGA) lipid-polymer hybrid nanoparticles (LPNs) on secretion of a) interleukin (IL)-6 and b) IL-8 from NuLi-1 bronchial epithelial cells (BECs). IL-6 and IL-8 were measured in supernatants of NuLi-1 BECs exposed to DOTAP-modified PLGA LPNs loaded with dsDNA oligonucleotides as indicated for $24 \mathrm{~h}$. Lines represent best-fit linear regressions.

There was no significant correlation between secreted IL- 6 or IL- 8 concentrations and LPN concentrations $\left(\mathrm{R}^{2}=0.1441, \mathrm{p}=0.120\right)$ (figure 3); however, small increases in cytokine secretion by NuLi-1 cells exposed to $1 \mathrm{mg} \cdot \mathrm{mL}^{-1}$ LPNs were evident, but this was not statistically significant.

\section{Increased intracellular miR-17 expression in BEC cultures treated with miR-17-loaded LPNs}

Delivery of nanoparticles to epithelial cell cultures is frequently studied using subconfluent monolayers. However, as the superficial layer of epithelia in vivo consists of tightly packed cells, fully confluent cell cultures are also relevant. Hence, LPNs loaded with miR-17 mimics or NC\#1 control were administered to subconfluent (low density) and fully confluent NuLi-1 monolayers with a visible tight-junction phenotype (high density), and miR-17 was measured by quantitative reverse transcription-PCR $48 \mathrm{~h}$ later. Inadequate removal of uninternalised nanoparticles can lead to misleadingly high miRNA mimic expression values due to their unintended inclusion in the cell lysates. To measure only intracellular miR-17, a method designed to remove extracellular nanoparticles from the samples was used [21]. All BEC cultures had significantly increased miR-17 when transfected with miR-17-loaded LPNs compared with cultures transfected with NC\#1-loaded LPNs, except for low-density cultures incubated with the highest dose wherein miR-17 was substantially increased. The nebulised LPNs also effectively transfected NuLi BECs, leading to significantly increased levels of intracellular miR-17 under all conditions tested (figure 4).

\section{Knockdown of IL-8 in NuLi-1 BECs by miR-17-loaded LPNs}

Previous studies have shown that miR-17 mimics can knockdown LPS-induced IL- 8 by up to $50 \%$ in BECs $[14,31]$. Figure 5 shows the effect of miR-17-loaded LPNs on basal and LPS-induced IL-8 production from NuLi cells. The miR-17-loaded LPNs almost exclusively significantly decreased basal and LPS-stimulated IL-8 secretion compared with treatment with NC\#1-loaded LPNs in both low-density and high-density cultures. To test the effects of nebulisation on the efficacy of miR-17-loaded LPNs to knockdown IL-8, NuLi-1 BECs were treated with LPNs that were collected following nebulisation with the Aerogen Solo nebuliser. As before, significant decreases in basal and LPS-induced IL-8 production were evident in almost all of the conditions tested (figure 6). These results show that after nebulisation, the miR-17-loaded LPNs retain their ability to inhibit IL-8 secretion from BECs.

\section{Discussion}

The aims of this study were to engineer inhalable miR-17-loaded DOTAP-modified PLGA LPNs and to test their ability to inhibit the induced secretion of IL-8, which is the target of miR-17, in BECs. This follows our previous findings that miR-17 is downregulated in BECs upon long-term exposure to pro-inflammatory stimuli, which contributes to the upregulation of IL-8 [14]. miR-17 is a member of the miR-17-92 cluster. This is a cluster with a complex expression pattern in inflammation and it can be contextually upregulated or downregulated in a cell type-dependent manner [14, 32]. A published protocol was adapted to prepare nanoparticles with properties previously considered suitable for delivery of miRNA mimics to the bronchial epithelium [17, 18, 22, 33]. Efficient delivery of nanoparticles to the airway epithelium depends on several factors. It is known that the mucus permeability is almost completely 

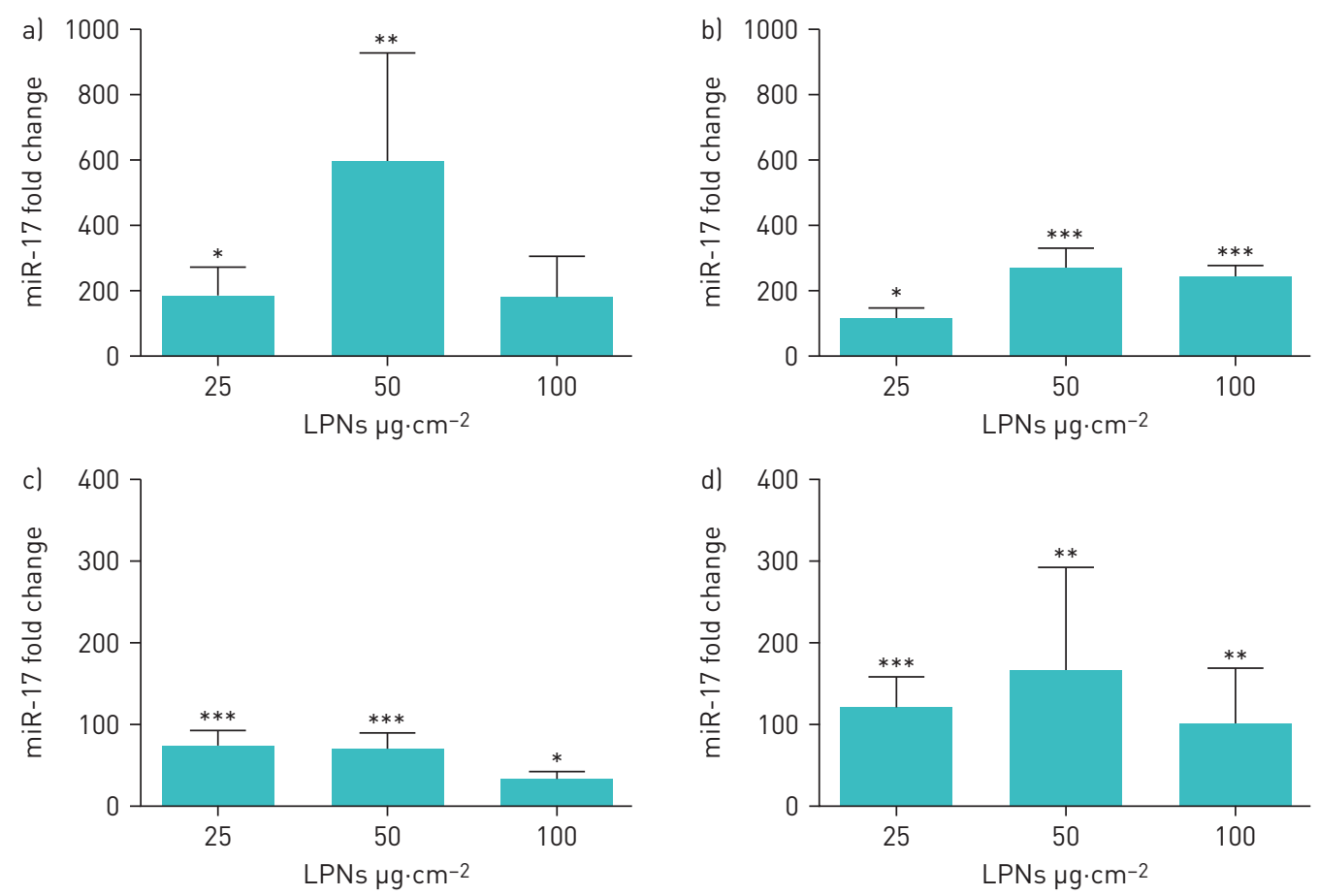

FIGURE 4 Change in intracellular concentration of microRNA (miR)-17 in NuLi-1 bronchial epithelial cells (BECs) treated with miR-17-loaded lipid-polymer hybrid nanoparticles (LPNs). LD: low density (NuLi-1 BECs cultured to subconfluent density); HD: high density (NuLi-1 BECs cultured at confluent density). a-d) The concentration of miR-17 was measured by quantitative reverse transcription-PCR in a, c) LD and b, d) HD NuLi-1 BECs transfected with $a, b)$ unnebulised or $c$, d) nebulised miR-17 LPNs at varying concentrations and compared with the concentration in BECs transfected with control LPNs. Data are presented as mean \pm SD. ${ }^{*}: p \leqslant 0.05 ;{ }^{* *}: p \leqslant 0.01 ;{ }^{* * *}: p \leqslant 0.001$, two-tailed t-test. $n=4$.

inhibited for particles $>300 \mathrm{~nm}[22,33]$, while nanoparticle endocytosis is considered most efficient at the $50-500 \mathrm{~nm}$ range $[34,35]$. Larger particles are usually phagocytosed by lung macrophages [24]. The size of the LPNs prepared in this study was within the $200-250 \mathrm{~nm}$ range. Their $\zeta$-potential was within the 15-45 $\mathrm{mV}$ range deemed optimal for nucleic acid delivery, and represents a compromise between maximal colloidal stability and minimal inflammatory stimulation $[9,36]$. The loading capacity, yield and encapsulation efficiency measurements were comparable to those found in other studies [35].

The miR-17-loaded LPNs in this study successfully delivered miR-17 to the BECs. miR-17 levels were substantially higher in all treated cultures; however, a linear dose-response relationship was not observed. Endocytosis, intracellular trafficking, endosomal escape and cargo release of LPNs are complex processes that may have an influence on dose kinetics, but examining this is beyond the scope of the current study. The delivery of miR-17 led to knockdown of IL-8 under both basal and pro-inflammatory conditions in subconfluent and confluent epithelial cell monolayers, exceeding the performance of previous cationic lipid-based transfection reagents [14].

Cytotoxicity profiling of DOTAP-modified PLGA LPNs in cell cultures has previously resulted in conflicting reports, showing that at high concentrations these LPNs can exert some toxicity [8, 17]. Based on cell viability and apoptosis measurements we did not observe any significant cytotoxic effects induced by the PLGA:DOTAP component of LPNs at the concentrations tested in NuLi-1 BECs, including the highest LPN concentration. The LPNs did not induce IL-6 and IL-8 except at $1 \mathrm{mg} \cdot \mathrm{mL}^{-1}$, a concentration that exceeds the range used for further functional experiments and exceeds the expected physiological dose for in vivo application. However, it is important to note that when loaded with miR-17 mimic, the basal levels of IL- 8 secreted by LPN-treated NuLi-1 cells was approximately 1.5-3.5-fold elevated compared with when the LPNs were loaded with dsDNA cargo. This observation deserves greater scrutiny in future studies.

Nebulisation of nanoparticles in 1-5 $\mu \mathrm{m}$ droplets has been demonstrated previously as an effective method to deliver nanoparticles to the pulmonary epithelium in vivo [37]. From in vitro experiments, we found that nebulisation of the miR-17-loaded LPNs reconstituted in $0.9 \%$ saline resulted in an aerosol with aerodynamic properties that could allow successful deposition of the LPNs in the secondary bronchi and 

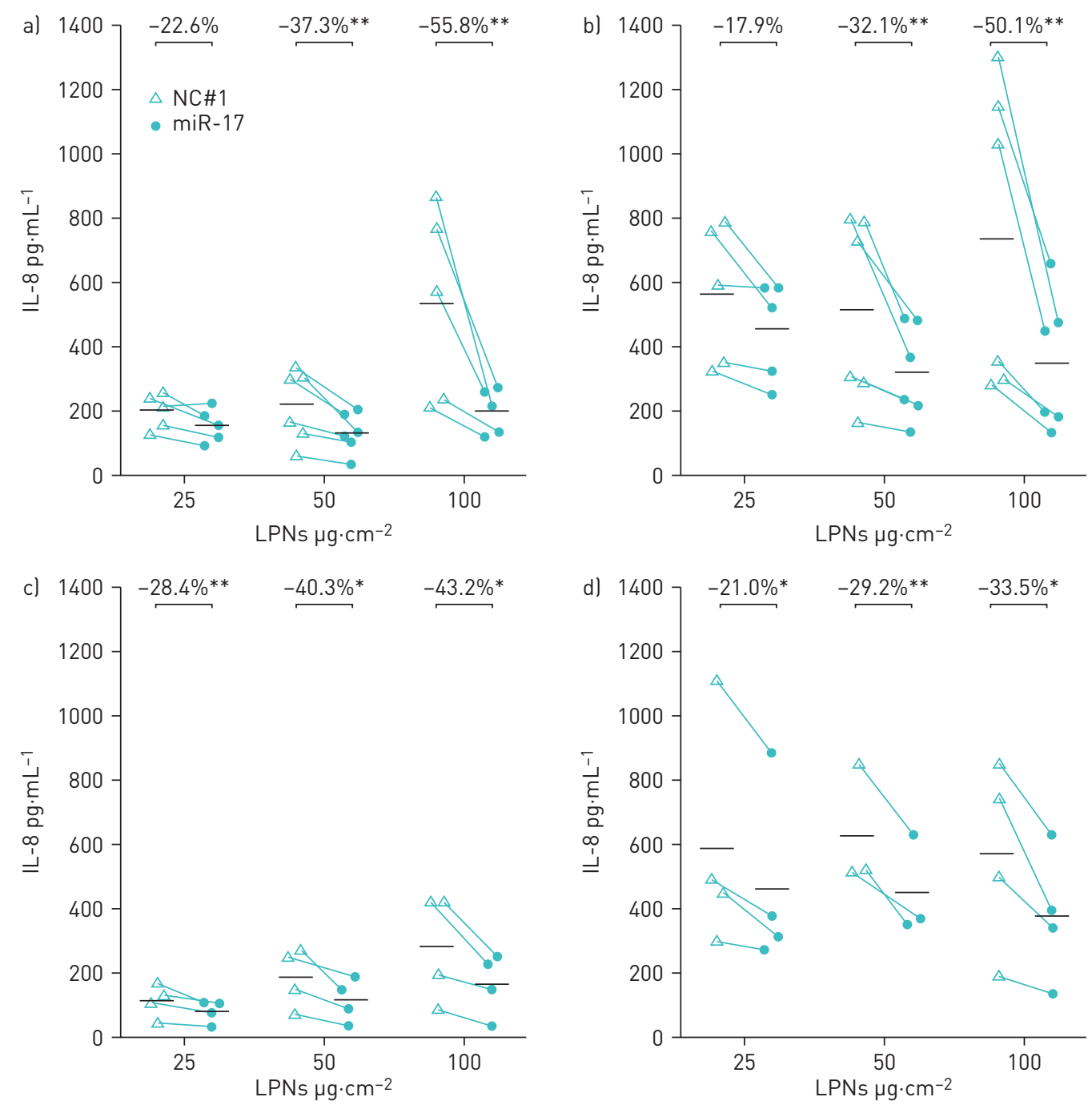

FIGURE 5 Effect of microRNA (miR)-17-loaded lipid-polymer hybrid nanoparticles (LPNs) on interleukin (IL)-8 expression. LPS: lipopolysaccharide; BEC: bronchial epithelial cell; LD: low density (NuLi-1 BECs cultured to subconfluent density); HD: high density (NuLi-1 BECs cultured at confluent density). a, c) Baseline and b, d) LPS-stimulated IL-8 expression in a, b) LD and c, d) HD NuLi-1 BEC cultures exposed to 25, 50 and $100 \mu \mathrm{g} \cdot \mathrm{cm}^{-2}$ miR-17-loaded LPNs or NC\#1-loaded LPNs. Individual data points are shown; bars represent mean values. ${ }^{*}: p \leqslant 0.05 ;{ }^{* *}: p \leqslant 0.01$, one-tailed ratio t-test. $n=6$ for $L D ; n=4$ for HD.

bronchioles upon inhalation. Future in vivo testing may confirm this. Shear forces from nebulisation may affect the LPNs and their cargo. However, our study shows that nebulisation of miRNA mimic-loaded PLGA LPNs does not affect their physicochemical properties or their transfection efficiency, indicating that the Aerogen Solo nebuliser is a suitable device for LPN delivery to the lungs. This also corroborates previous findings for the nebulisation of plasmid DNA-loaded PLGA LPNs [37]. Nonetheless differences were evident in the transfection efficiencies of the nebulised versus native LPNs and further research would be required in order to understand why this difference exists.

This study also presents nanoparticle counting using the Nanosight NS300 as a novel and effective method to quantify nanoparticles directly by visualisation and counting after their retrieval from cascade impactors. However, it is important that the nanoparticle concentrations in suspension be normalised to within a range that can be quantified accurately using the Nanosight NS300. We propose that this method is a valid complement to existing methods of reproducibly quantifying nanoparticles in suspension.

This study has some limitations. In the absence of in vivo testing, these results merely represent proof-of-concept efficacy of miRNA-loaded LPNs in vitro and could only be performed in a single cell line. Examination of the behaviour of the LPNs in mucus-producing air-liquid interface cultures or their effects on other miR-17 targets, such as STAT3 (signal transducer and activator of transcription 3), Mcl-1 

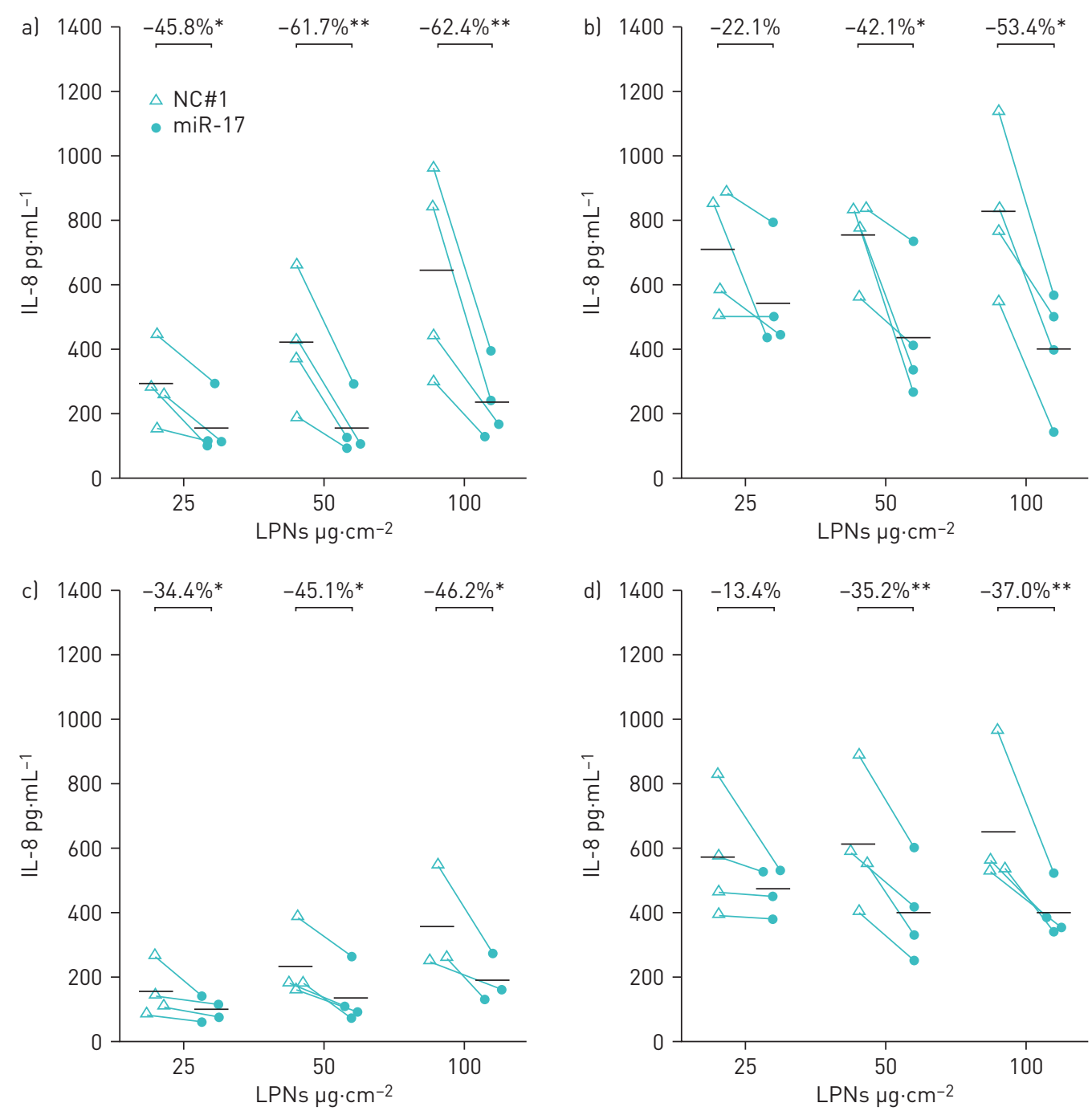

FIGURE 6 Effect of nebulised microRNA (miR)-loaded lipid-polymer hybrid nanoparticles (LPNs) on interleukin (IL)-8 expression. LPS: lipopolysaccharide; BEC: bronchial epithelial cell; LD: low density (NuLi-1 BECs cultured to subconfluent density); HD: high density (NuLi-1 BECs cultured at confluent density). a, cl Baseline and b, d) LPS-stimulated IL-8 expression in $a, b)$ LD and c, d) HD NuLi-1 BEC cultures exposed to 25,50 and $100 \mu \mathrm{g} \cdot \mathrm{cm}^{-2}$ nebulised miR-17-loaded LPNs or NC\#1-loaded LPNs. Individual data points are shown; bars represent mean values. ${ }^{*}: p \leqslant 0.05 ;{ }^{* *}: p \leqslant 0.01$, one-tailed ratio t-test. $n=6$ for LD; $n=4$ for HD.

(myeloid cell leukaemia 1) and TXNIP (thioredoxin interacting protein) [38, 39], was beyond the scope of the current investigation.

Inflammatory lung diseases are among the most common lung pathologies. A chronic oversecretion of IL-8 by airway epithelia and leukocytes and consequent neutrophilia are common symptoms of COPD, CF, infection and certain types of asthma [5]. Neutrophilia in response to high IL-8 levels is a compounding problem that results in progressive degeneration and remodelling of lung tissue. BECs are important immune-modulating cells and represent the first line of defence against airway insult, but they are particularly sensitive to upregulated neutrophil protease activity. Beyond IL-8, there are other validated pro-inflammatory human targets of miR-17 that are expressed by BECs, e.g. ICAM1 (intercellular adhesion molecule 1) and TGF $\beta$ R2 (transforming growth factor- $\beta$ type II serine/threonine kinase receptor). These factors can contribute to infectious and inflammatory lung disease, including rhinovirus infection, CF, asthma and COPD, and therefore the approach under investigation here could have therapeutic potential for those conditions [40-42]. This proof-of-concept study provides evidence that DOTAP-modified PLGA LPNs are suitable, nontoxic carriers for nebulised delivery of miR-17 to BECs and forms a platform upon which future miRNA-based therapeutics could be developed for inflammatory lung disease. 
Acknowledgements: The authors would like to thank the staff at Aerogen Ltd (Galway, Ireland) for their support and advice for the nebulisation experiments, and the students and staff members of C. Foged's laboratory at the Dept of Pharmacy, University of Copenhagen (Copenhagen, Denmark) who provided consulting to this study. The authors would like to acknowledge Joseph Zabner (University of Iowa, Carver College of Medicine, Iowa City, IA, USA) for providing the NuLi-1 cell line used in this study.

Conflict of interest: S. Vencken reports grants from Science Foundation Ireland, during the conduct of the study. C. Foged has nothing to disclose. J.M. Ramsey has nothing to disclose. L. Sweeney has nothing to disclose. S-A. Cryan has nothing to disclose. R.J. MacLoughlin has nothing to disclose. C.M. Greene reports grants from the National Children's Research Centre and Cystic Fibrosis Foundation Therapeutics during the conduct of the study.

Support statement: Funding for this work is gratefully acknowledged from the National Children's Research Centre (C/13/1 to C.M. Greene), Cystic Fibrosis Foundation Therapeutics (GREENE15XXO), a Science Foundation Ireland Industrial Fellowship (13/IA/1840 to S. Vencken) and a Science Foundation Ireland Investigators Program (13/IA/1840 to S-A. Cryan and J.M. Ramsey). Funding information for this article has been deposited with the Crossref Funder Registry.

\section{References}

1 Barnes PJ. Immunology of asthma and chronic obstructive pulmonary disease. Nat Rev Immunol 2008; 8: $183-192$.

2 Greene CM, McElvaney NG. Toll-like receptor expression and function in airway epithelial cells. Arch Immunol Ther Exp 2005; 53: 418 .

3 Greene CM, Carroll TP, Smith SGJ, et al. TLR-induced inflammation in cystic fibrosis and non-cystic fibrosis airway epithelial cells. J Immunol 2005; 174: 1638-1646.

4 Nakamura H, Yoshimura K, McElvaney NG, et al. Neutrophil elastase in respiratory epithelial lining fluid of individuals with cystic fibrosis induces interleukin-8 gene expression in a human bronchial epithelial cell line. J Clin Invest 1992; 89: 1478-1484

5 Pease JE, Sabroe I. The role of interleukin-8 and its receptors in inflammatory lung disease. Am J Respir Med 2002; 1: 19-25.

6 Yao H, de Boer WI, Rahman I. Targeting lung inflammation: novel therapies for the treatment of COPD. Curr Respir Med Rev 2008; 4: 57-68.

7 Merkel OM, Rubinstein I, Kissel T. siRNA delivery to the lung: what's new? Adv Drug Deliv Rev 2014; 75 $112-128$

8 Jensen DK, Jensen LB, Koocheki S, et al. Design of an inhalable dry powder formulation of DOTAP-modified PLGA nanoparticles loaded with siRNA. J Control Release 2012; 157: 141-148.

9 Jensen DMK, Cun D, Maltesen MJ, et al. Spray drying of siRNA-containing PLGA nanoparticles intended for inhalation. J Control Release 2010; 142: 138-145.

10 Conti DS, Brewer D, Grashik J, et al. Poly(amidoamine) dendrimer nanocarriers and their aerosol formulations for siRNA delivery to the lung epithelium. Mol Pharm 2014; 11: 1808-1822.

11 Kusumoto K, Akita H, Ishitsuka T, et al. Lipid envelope-type nanoparticle incorporating a multifunctional peptide for systemic siRNA delivery to the pulmonary endothelium. ACS Nano 2013; 7: 7534-7541.

12 Bartel DP. MicroRNAs: genomics, biogenesis, mechanism, and function. Cell 2004; 116: 281-297.

13 Broderick JA, Zamore PD. MicroRNA therapeutics. Gene Ther 2011; 18: 1104-1110.

14 Oglesby IK, Vencken SF, Agrawal R, et al. miR-17 overexpression in cystic fibrosis airway epithelial cells decreases interleukin-8 production. Eur Respir J 2015; 46: 1350-1360.

15 Vij N, Min T, Marasigan R, et al. Development of PEGylated PLGA nanoparticle for controlled and sustained drug delivery in cystic fibrosis. J Nanobiotechnology 2010; 8: 22.

16 Pirooznia N, Hasannia S, Lotfi AS, et al. Encapsulation of alpha-1 antitrypsin in PLGA nanoparticles: in vitro characterization as an effective aerosol formulation in pulmonary diseases. J Nanobiotechnology 2012; 10: 20.

17 Colombo S, Cun D, Remaut K, et al. Mechanistic profiling of the siRNA delivery dynamics of lipid-polymer hybrid nanoparticles. J Control Release 2015; 201: 22-31.

18 Cun D, Jensen DK, Maltesen MJ, et al. High loading efficiency and sustained release of siRNA encapsulated in PLGA nanoparticles: quality by design optimization and characterization. Eur J Pharm Biopharm 2011; 77: $26-35$.

19 Zabner J, Karp P, Seiler M, et al. Development of cystic fibrosis and noncystic fibrosis airway cell lines. Am Physiol Lung Cell Mol Physiol 2003; 284: L844-L854.

20 Miller SDW, Greene CM, McLean C, et al. Tauroursodeoxycholic acid inhibits apoptosis induced by Z alpha-1 antitrypsin via inhibition of Bad. Hepatology 2007; 46: 496-503.

21 Colombo S, Nielsen HM, Foged C. Evaluation of carrier-mediated siRNA delivery: lessons for the design of a stem-loop qPCR-based approach for quantification of intracellular full-length siRNA. J Control Release 2013; 166 $220-226$.

22 Lai SK, Wang Y-Y, Hanes J. Mucus-penetrating nanoparticles for drug and gene delivery to mucosal tissues. Adv Drug Deliv Rev 2009; 61: 158-171.

23 Moore TL, Rodriguez-Lorenzo L, Hirsch V, et al. Nanoparticle colloidal stability in cell culture media and impact on cellular interactions. Chem Soc Rev 2015; 44: 6287-6305.

24 Champion JA, Walker A, Mitragotri S. Role of particle size in phagocytosis of polymeric microspheres. Pharm Res 2008; 25: 1815-1821.

25 Mura S, Hillaireau H, Nicolas J, et al. Influence of surface charge on the potential toxicity of PLGA nanoparticles towards Calu-3 cells. Int J Nanomedicine 2011; 6: 2591-2605.

26 Dandekar P, Venkataraman C, Mehra A. Pulmonary targeting of nanoparticle drug matrices. J Aerosol Med Pulm Drug Deliv 2010; 23: 343-353.

27 Kelly C, Yadav AB, Lawlor C, et al. Therapeutic aerosol bioengineering of siRNA for the treatment of inflammatory lung disease by TNF $\alpha$ gene silencing in macrophages. Mol Pharm 2014; 11: 4270-4279. 

therapeutics for systemic delivery. Adv Drug Deliv Rev 2006; 58: 1030-1060. Verma NK, Crosbie-Staunton K, Satti A, et al. Magnetic core-shell nanoparticles for drug delivery by nebulization. J Nanobiotechnol 2013; 11: 1.

30 Powell D, Chandra S, Dodson K, et al. Aptamer-functionalized hybrid nanoparticle for the treatment of breast cancer. Eur J Pharm Biopharm 2017; 114: 108-118.

$31 \mathrm{Yu}$ Z, Willmarth NE, Zhou J, et al. microRNA 17/20 inhibits cellular invasion and tumor metastasis in breast cancer by heterotypic signaling. Proc Natl Acad Sci USA 2010; 107: 8231-8236.

32 Tazi MF, Dakhlallah DA, Caution K, et al. Elevated Mirc1/Mir17-92 cluster expression negatively regulates autophagy and CFTR (cystic fibrosis transmembrane conductance regulator) function in CF macrophages. Autophagy 2016; 12: 2026-2037.

33 Suk JS, Kim AJ, Trehan K, et al. Lung gene therapy with highly compacted DNA nanoparticles that overcome the mucus barrier. I Control Release 2014; 178: 8-17.

34 Oh N, Park J-H. Endocytosis and exocytosis of nanoparticles in mammalian cells. Int J Nanomed 2014; 9 Suppl. 1, 51-63.

$35 \mathrm{Xu}$ A, Yao M, Xu G, et al. A physical model for the size-dependent cellular uptake of nanoparticles modified with cationic surfactants. Int J Nanomed 2012; 7: 3547-3554.

36 Feng S-S, Ruan G, Li Q-T. Fabrication and characterizations of a novel drug delivery device liposomes-in-microsphere (LIM). Biomaterials 2004; 25: 5181-5189.

37 Nguyen J, Reul R, Betz T, et al. Nanocomposites of lung surfactant and biodegradable cationic nanoparticles improve transfection efficiency to lung cells. J Control Release 2009; 140: 47-54.

38 Kumar R, Sahu SK, Kumar M, et al. MicroRNA 17-5p regulates autophagy in Mycobacterium tuberculosis-infected macrophages by targeting Mcl-1 and STAT3. Cell Microbiol 2016; 18: 679-691.

39 Coucha M, Mohamed IN, Elshaer SL, et al. High fat diet dysregulates microRNA-17-5p and triggers retinal inflammation: role of endoplasmic-reticulum-stress. World J Diabetes 2017; 8: 56-65.

$40 \mathrm{Bi} \mathrm{J}, \mathrm{Hu} \mathrm{Y}$, Peng Z, et al. Changes and correlations of serum interleukins, adhesion molecules and soluble E-selectin in children with allergic rhinitis and asthma. Pak J Med Sci 2018; 34: 1288-1292.

41 Zhou X, Zhu L, Lizarraga $\mathrm{R}$, et al. human airway epithelial cells direct significant rhinovirus replication in monocytic cells by enhancing ICAM1 expression. Am J Respir Cell Mol Biol 2017; 57: 216-225.

42 Dutta RK, Chinnapaiyan S, Rasmussen L, et al. A neutralizing aptamer to TGFBR2 and miR-145 antagonism rescue cigarette smoke- and TGF- $\beta$-mediated CFTR expression. Mol Ther 2019; 27: 442-455. 